\title{
Around the world and back again
}

\author{
Sherene Loi is a medical oncologist and clinician scientist at the Peter MacCallum Cancer Centre in Melbourne, \\ Australia. Her laboratory studies the cancer genome in breast cancer and its interface with the immune \\ microenvironment in order to develop more effective drugs and rational combinations.
}

\section{Sherene Loi}

W hen I was nine years old, my family traveled from Melbourne to Vancouver, Canada, to help care for my terminally ill uncle. He had been diagnosed with stage 4 colorectal cancer after what was initially thought to be a routine appendectomy. As I understand it, he was subsequently diagnosed with familial adenomatous polyposis, which is an inherited disorder predisposing individuals to colon cancer, and his extended family are now followed up for this. He died aged 35 , leaving his wife (my mother's sister) and three-year-old daughter behind. I still remember him showing me all these lumps on his abdomen, and although a young child does not understand the permanence of death, I certainly remember the prevailing sadness at that time.

Years on, I did a medical oncology rotation with a rather sardonic professor who always had a lot of words of advice for the aspiring medical oncology trainee. "Sherene," he would say, "the reason why the coffin lids are nailed on is to stop the medical oncologists getting their last dose of chemo in!" and "If you want a good next life, come back as a phase I or II trial as they are all positive." These words led me to understand that the field of oncology needed strong clinical acumen, more effective oncology drugs and to always question the validity of scientific data.

After completing my specialist training in Australia, I traveled across the world to Belgium to work with Martine Piccart, Christos Sotiriou and Laura Van't Veer. My project as a research fellow was working on bringing the MammaPrint multigene assay into the clinic. This was an exciting novel prognostic biomarker developed from the new whole-genome microarrays that promised to identify which early-stage breast cancer patients did not need chemotherapy based on their biology. Along with the waffles, chocolate and beers that are characteristic of Belgian life, were many inspiring years: new genomic technologies were emerging, allowing us access for the first time to the cancer genome, as well as an understanding of relevant targets in

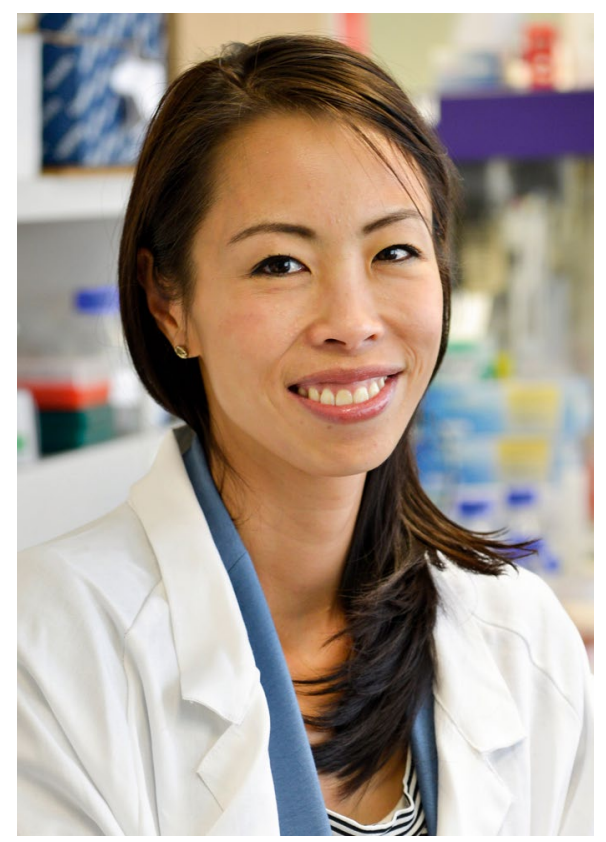

Credit: Sherene Loi

breast cancer. We were very excited by the new HER2-targeted agentstrastuzumab, pertuzumab and lapatinibwhich promised, and have subsequently been transformative for, extending survival of these patients. My European experience taught me the power of multidisciplinary teams, international collaboration, translational research and the importance of a vision for achieving better patient outcomes.

During that time I began collaborating with pathologist Roberto Salgado and biostatistician Stefan Michiels. We have since done extensive research on how the immune system reacts to breast cancer, how to quantify this, and understanding its relevance for breast cancer prognosis and response to immunotherapies. We work well together: my eternal optimism about every hypothesis I generate with the analytical robustness of a pathologist and the cynicism of a statistician about every result. The end product of this collaboration is reproducible science.
Today I am back in Melbourne, and while Australia is far away from Europe, the world is now well connected, which makes being in touch with the rest of the world easier. I have a busy lab and clinic, and also lead the breast cancer clinical trials unit, which forms the circle of translation. Caring for patients on clinical trials is an important part of what I do, as I can get an early feel for whether a drug has promising anti-tumor activity, and, ultimately, patients remind you why you are there. I am also a mother to two busy teen children. My advice to current aspiring female researchers who are new mothers is to push on and stay focused on producing good research as the busy period involving coordinating child care, including pickups and drop-offs, and sleep deprivation eventually does end.

A big part of my research uses technology, which now has incredible resolution, to ask new questions and revisit old ones from a different angle. Having well-annotated patient samples from clinical trials also provides an important basis to understand mechanism and resistance of new agents, and to develop robust biomarkers for patient care. Today's research is far more complex than before, with large volumes of data being produced, and I believe that teams comprising people from multiple disciplines are essential to move successfully in the circle of preclinical research and back to the patients. For breast cancer patients, much has been achieved in the last 20 years in the quest for personalized and more effective medicine, and I hope that I can contribute to further improvements in survival with this still deadly disease.

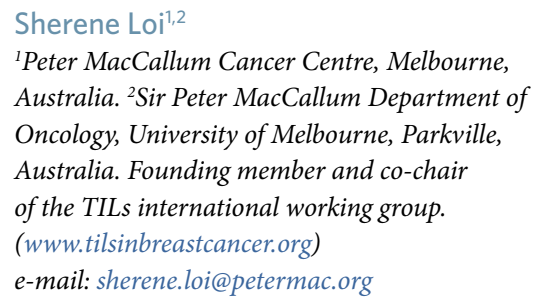

Published online: 7 October 2019

https://doi.org/10.1038/s41591-019-0578-0 